\title{
RECUENTOS CROMOSOMICOS EN ZORNIA, STYLOSANTHES y DESMODIUM (LEGUMINOSAE)
}

por RICARDO VANNI*

\section{Summary}

Chromosome numbers of three species of Zornia, three of Desmodium and one of Stylosanthes are reported for the first time. Confirmation of a previous count for Desmodium leiocarpum is included.

La tribu Hedysareae (Leguminosae), está representada en el país por los géneros Poiretia, Aeschynomene, Discolobium, Desmodium, Stylosanthes, Amicia, Nissolia, Zornia, Ornithopus, Chaetocalyx y Arachis. En esta oportunidad se dan a conocer por primera vez recuentos cromosómicos de tres especies de Zornia, tres de Desmodium y una de Stylosanthes. Además se confirma el guarismo publicado para Desmodium leiocarpum.

El material estudiado figura en el Cuadro I; los ejemplares testigos fueron identificados por el autor y se encuentran depositados en el herbario del IBONE (CTES).

Para los recuentos se usaron células madres de polen y raicillas de semillas germinadas en laboratorio, fijándose ambas en 3:1 (alcohol absoluto-ácido acético), manteniéndose luego en alcohol 70, en refrigerador; las raicillas fueron pretratadas con 8--oxiquinoleína. Para coloración se usó la técnica de Feulgen, macerando posteriormente en una gota de orccína lactoacética modificada (Mroginski y Fernández, 1979).

* Profesor Titular Interino de Botánica Sistemática y Fitogeografía, Facultad de Ciencias Agrarias (UNNE), Corrientes. Trabajo realizado con una Beca de Formación Superior del Consejo Nacional de Investigaciones Científicas y Técnicas. 


\section{Cuadro I}

\begin{tabular}{|c|c|c|c|c|}
\hline Especie & $2 n$ & Fig. & Procedericia & Coleccionista \\
\hline $\begin{array}{l}\text { Desmodium pachyrhizum } \\
\text { Vog. }\end{array}$ & 22 & 6 & $\begin{array}{l}\text { Corrientes, Dpto. } \\
\text { Ituzaingó, río Agua- } \\
\text { pey y R. } 38\end{array}$ & T. 1691 \\
\hline $\begin{array}{l}\text { Desmodium glabrum } \\
\text { (Mill.)DC. }\end{array}$ & 22 & 5 & $\begin{array}{l}\text { Bolivia, Tarija } \\
\text { Pto. Margarita }\end{array}$ & K. $391: 5$ \\
\hline $\begin{array}{l}\text { Desmodium riedelii } \\
\text { (Schindl.)Burk. }\end{array}$ & $11 \mathrm{II}$ & 7 & $\begin{array}{l}\text { Corrientes, Dpto. } \\
\text { Capital, ayo. Ria- } \\
\text { chuelo }\end{array}$ & V. 457 \\
\hline $\begin{array}{l}\text { Desmodium leiocarpum } \\
\text { (Spreng.) Don }\end{array}$ & 11 II & 8 & $\begin{array}{l}\text { Brasil, Paraná, BR } \\
277 \mathrm{Km} 477\end{array}$ & F. 360 \\
\hline $\begin{array}{l}\text { Stylosanthes macrosoma } \\
\text { Blake }\end{array}$ & 20 & 4 & $\begin{array}{l}\text { Corrientes, Dpto. } \\
\text { Capital, Molina Punta }\end{array}$ & V. 436 \\
\hline $\begin{array}{l}\text { Zornia gemella (Willd.) } \\
\text { Vog. }\end{array}$ & 20 & 1 & $\begin{array}{l}\text { Corrientes, Dpto. } \\
\text { Mércedes, Mercedes }\end{array}$ & T. 2428 \\
\hline $\begin{array}{l}\text { Zornia multinervosa } \\
\text { Burk. ex Bacig. }\end{array}$ & 20 & 3 & $\begin{array}{l}\text { Corrientes, Dpto. } \\
\text { San Martín, Tres } \\
\text { Cerros }\end{array}$ & S. 21868 \\
\hline Zornia reticulata $\mathrm{Sm}$. & 20 & 2 & $\begin{array}{l}\text { Jujuy, Dpto. El Car- } \\
\text { men R. } 66 \text { y río Perico }\end{array}$ & S. 22314 \\
\hline
\end{tabular}

Las abreviaturas de los coleccionistas son: F: Silvia Ferrucci, K: Antonio Krapovickas, S: Aurelio Schinini, T: Sara Tressens, V: Ricardo Vanni.

Los recuentos realizados en Zornia gemella, Z. reticulata y $Z$. multinervosa, todas con $2 \mathrm{n}=20$ cromosomas, concuerdan con los ya publicados para otras especies dei género (Krapovickas

Fig. 1-6. Cromosomas somáticos. 1, Zornia gemella $(2 \mathrm{n}=20) ; 2$, Zornia reticulata $(2 \mathrm{n}=20) ; 3$, Zornia multinervosa $(2 \mathrm{n}=20) ; 4$, Stylosanthes macrosoma $(2 \mathrm{n}=20) ; 5$, Desmodium glabrum $(2 \mathrm{n}=22) ; 6$, Desmodium pachyrhizum $(2 \mathrm{n}=22)$. Fig. 7-8. Cromosomas meióticos. 7, Desmodium riedelii MI (11 II); 8, Desmodium leiocarpum MI (11 II). Todos X 2000. 


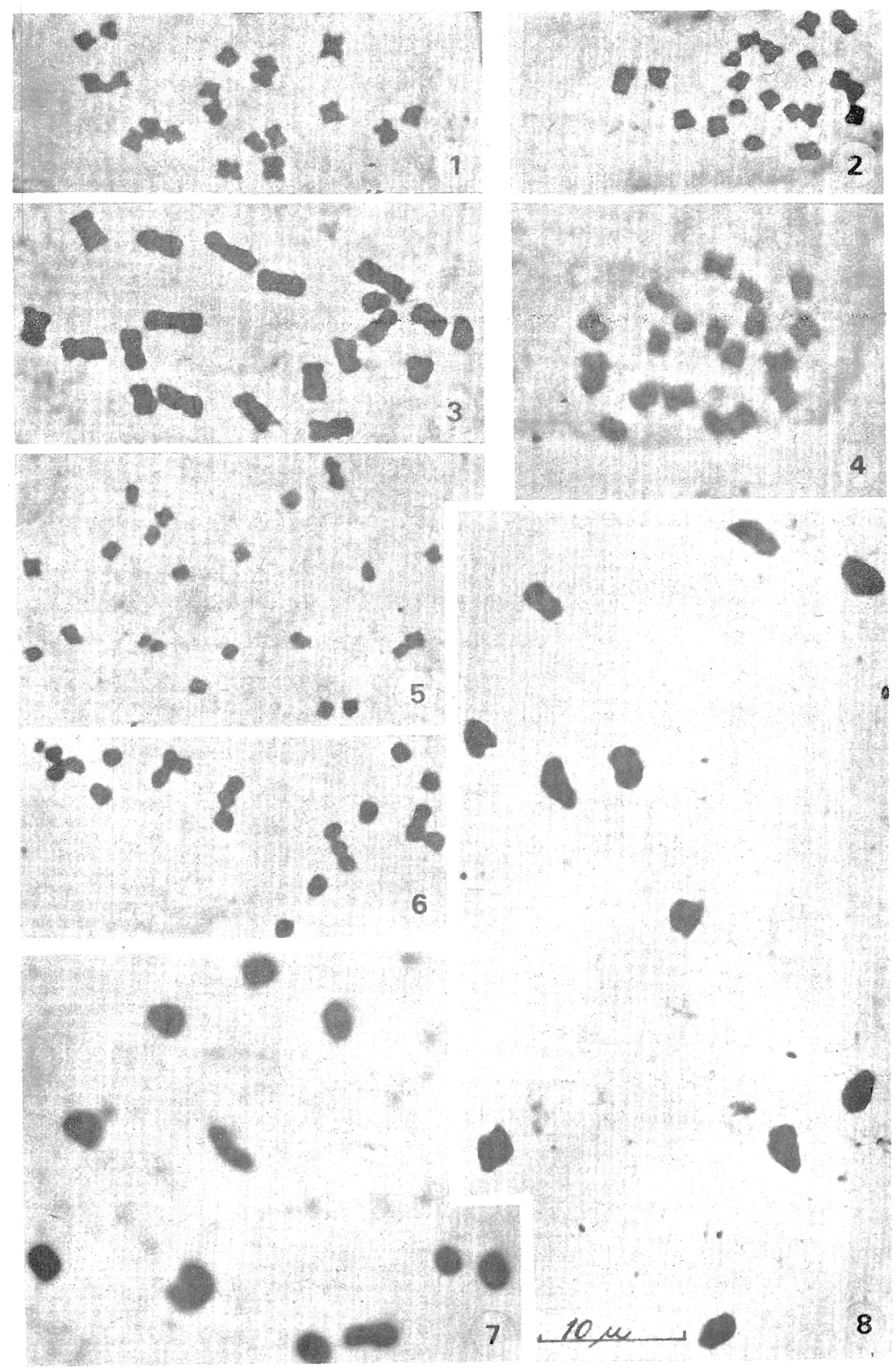


1965; Coleman, J. and De Menezes, 1980; Vanni, 1983). La diferencia de tamaño de los cromosomas de $Z$. multinervosa, con respecto a los de las otras dos especies, que se observan en la lámina, se debería a diferentes tiempos de pretratamiento, dos horas en la primera especie y tres horas, en las dos restantes.

Desmodium glabrum, $2 \mathrm{n}=22$; que vive en Argentina, es del grupo de especies con plantas de gran crecimiento vegetativo, utilizables como forrajeras. Desmodium riedelii, $2 \mathrm{n}=11$ II, es una de las dos especies del país con artejos del fruto muy grandes ( $\pm 1 \mathrm{~cm}$ long.). Fue coleccionada únicamente en Corrientes y en su localidad tipo, Rio Pardo, Brasil. Desmodium pachyrhizum, era la única especie con hojas reducidas a un folíolo, de las cuatro que crecen en Argentina, que no había sido estudiada cariológicamente; el número hallado, $2 n=22$, coincide con el de las otras tres especies de este grupo. De las especies detalladas estos serían los primeros registros. El guarismo para D. leiocarpum, $2 \mathrm{n}=11$ II, concuerda con el ya publicado por FrahmLeliveld (1953).

Agradezco al Ing. Agr. Aveliano Fernández, su valiosa colaboración.

Bibliografía

Burkart, 4. 1939. Las Leguminosas Hedysareas de la República Argentina y Regiones Adyacentes. Darwiniana 3: 117 . 301 .

Coleman, J. and De Menezes, E. 1980. Chromosome numbers in Leguminosae, from state of São Paulo, Brazil, Rhodora 82(831): $475-481$.

Fedorov, 1974. Chromosome numbers of flowering plants. 1926. 
Frahm-Leliveld. 1953. Some chromosome numbers in tropical leguminous plants. Fuphytica 2(1): 46-48.

Goldblatt, P. 1981. Advances in Legume Systematics, ed. R. M. Polhil \& P.M. Raven. 427-463.

-_-_-1981. Monograph in Systematics Botany from Missouri Botanical Garden Vol.5. Index to plant chromosome numbers 1975-1978.

- - - 1984. Monograph in Systematics Botany from the Missouri Botanical Garden Vol. 8. Index to plant chromosome numbers 1979-1981 .

Krapovickas, A. 1965. Recuentos cromosómicos de Leguminosas. Kurtziana 2: 91 -94.

Mohlenbrock, R.H. 1961. A monograph of the Leguminous genus Zornia . Webbia 16(1): 1-143.

Mroginski, L.A. y Fernández, A. 1979. Cultivo in-vitro de an teras de especies de Arachis (Leguminosae). Oleagineux 34.(5): $243-248$.

Vanni, R.O. 1983. Cromosomas de Hedysareae. Bonplandia $5(24): 227-233$. 